\title{
RPM Variable Solenoid Engine
}

\author{
Navdeep Singh ${ }^{1}$, Abdul Sami Shaik Abdul Khadeer ${ }^{2} \&$ Mohammed Sameer Baig $^{3}$ \\ ${ }^{1,2}$ Student, ${ }^{3}$ Faculty In-Charge, Level 3 Diploma in Engineering, ASTI Academy, Dubai, UAE.
}

DOI: http://doi.org/10.46382/MJBAS.2021.5401

Copyright: (๑) 2021 Navdeep Singh et al. This is an open access article distributed under the terms of the Creative Commons Attribution License, which permits unrestricted use, distribution, and reproduction in any medium, provided the original author and source are credited.

\section{ABSTRACT}

The solenoid engine project works on electromagnetic principles of a solenoid. A solenoid is copper coil. The wire is tightly folded multiples to form a solenoid coil. When electrical charge is supplied to the solenoid, it creates a magnetic flux as the current passes through it. The solenoid engine is $V 4$ engine. There are four solenoids as cylinders and there is one piston in each solenoid. The metal pistons inside the solenoids react to the magnetic field created by the solenoids when current is flowing through. When the electrical current is no more flowing through the solenoid, the pistons fall back down to their original position due to gravity. As a result, the piston moves in a linear motion. The pistons are connected to the crankshaft. When the pistons move in a specific sequence, the crankshaft rotates. The linear motion has been converted to rotary motion using this mechanism. The RPM of the engine was controlled by adding a potentiometer to control the resistance of voltage provided to the solenoids. Therefore, controlling how fast the engine operates.

Keywords: Electromagnet, Magnetic flux, Potentiometer, RPM, Solenoid.

\section{Introduction}

A solenoid is typically a coil of wire that emits electromagnetic properties when carrying an electric current. The term "solenoid" was invented in the late 1820 s by a French physicist named Andre-Marie Ampere. A solenoid engine is simply based on the electromagnetic properties of the solenoid. The solenoid engine was invented by Thomas Davenport and Joseph Henry in July 1834. Solenoid engines are a possible alternative to an internal combustion engine in light vehicles like bikes. Although generally they do not produce a lot of power, there are few ways to increase the electromagnetic force produced. The number of turns in the coil can be increased and the current in the coil can also be increased.

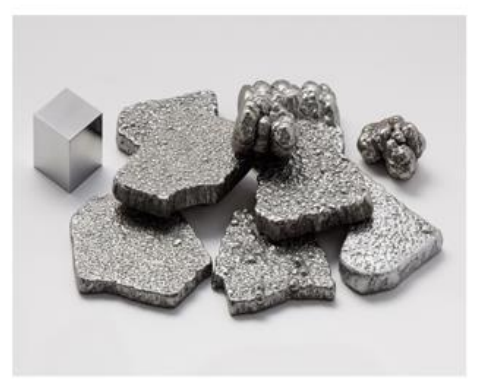

Fig.1. Metals

The metal piston inside the solenoid coil moves back and forth due to electromagnetism and causes the crankshaft to rotate. A solenoid engine can be similar to an internal combustion engine. Both engine's purpose is to transfer the linear motion of the pistons to rotary motion of the crank. The basic mechanism is almost identical. However, there are few major differences. Unlike the internal combustion engines, the solenoid engine does not rely on flammable fuels to operate. For this reason, they do not require complex fuel injectors and spark plugs to initiate a combustion. Therefore, it is arguably eco-friendlier. 


\section{Materials Used in the Project}

\subsection{Metals}

Metal is a material that, when freshly prepared, polished or broken, shows a shiny appearance and relatively good electrical and thermal conductivity. Metals are generally malleable (which means it can be hammered into thin metal sheets) or ductile (which means it can be drawn into thin wires). Metal can be a chemical element, such as iron; an alloy, such as stainless steel; or a molecular compound, such as Poly Sulphur Nitride. In physics, a metal is generally considered to be any substance that can conduct electricity at absolute zero temperature. Many compounds and elements which are normally not classified as metals, turn into metals at higher pressure. For example, non-metallic iodine gradually becomes metallic at a pressure 40 to 170,000 times atmospheric pressure. Likewise, some materials that are considered metals can also become non-metals. For example, sodium becomes non-metallic at a pressure 2 million times lower than atmospheric pressure.

\subsection{Properties of metals: Structure and form}

Metals are generally ductile, and will deform under load without splitting. It is believed that the non-directionality of metal bonds is the main contributor to the ductility of most metal solids. In contrast, in ionic compounds such as table salt, when the planes of ionic bonds slide across each other, the resulting positional changes will move ions with the same charge to nearby, causing crystals to split. This shift is not observed in covalently bonded crystals (such as diamond), where cracks and crystal fragmentation occur. Reversible elastic deformation in metals can be described by Hooke's law of restoring force, where stress and strain become linearly proportional.

\subsection{Properties of metals: Electrical and Thermal properties}

Metals are relatively good electrical conductors due to their electronic structure. The electrons in a substance can only have a fixed energy level instead of a variable energy level. In the case of metals, the energy level of the electrons in the electron cloud corresponds to the energy level at which electrical conduction can at least partially take place. In semiconductors such as silicon or non-metals such as sulfur there is an energy gap between the electrons in the substance and the energy level at which conduction can take place. Resulting non - metals and semiconductors to be relatively bad conductors.

\subsection{Properties of metals: Chemical Properties}

Metals are usually prone to forming attacks through the loss of electrons. The bulk will react with oxygen present in the air to form certain oxides in different time scales (potassium will burn within seconds; oxidized iron will take years). Some others, such as Palladium, Platinum and Gold, do not react at all with the atmosphere. Metal oxides are generally fundamental, unlike those of non-metals, which are acid or neutral. Exclusions are oxides with higher oxidation states, such as Chromium trioxide, Dimanganese heptoxide and Osmium tetroxide, which have purely acidic reactions.

\subsection{Metals used in the project: Iron}

Iron is a chemical element with the symbol Fe (from Latin: Ferrum) and the atomic number 26. It is a metal 
belonging to the first transition series and the eighth group of the periodic table. It is the most abundant element on earth, second only to oxygen (32.1\% and $30.1 \%$ respectively), and constitutes a large part of the outer core and inner core of the earth. It is the fourth most ample element in the earth's crust.

\subsection{Metals used in the project: Copper}

Copper is a chemical element, its symbol is $\mathrm{Cu}$, and its atomic number is 29 . It is a soft, ductile metal with very high thermal and electrical conductivity. The newly exposed surface made of pure copper is pinkish orange. Copper is used as a conductor of heat and electricity, as a component of building materials and various metal alloys, for example for temperature measurement.

\subsection{Metals used in the project: Acrylic Plastic}

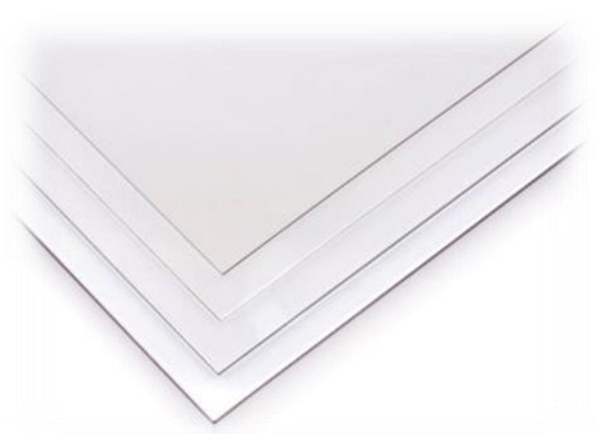

Fig.2. Acrylic Plastic

Acrylic is a transparent plastic with excellent strength, rigidity and optical transparency. Acrylic sheets are easy to make, adhere well with adhesives and solvents, and are easy to thermoform. Compared to many other transparent plastics, it has excellent weather resistance.

Acrylic glass has properties similar to glass - clarity, brilliance and transparency - but it weighs half that of glass and its impact resistance is many times higher than that of glass. From durable signs and skylights to eye-catching store fixtures, display shelves, and shelving, acrylic plastic has exceptional versatility, durability, and aesthetic qualities.

\section{Working Principle}

As mentioned earlier, the solenoid engine works on the electromagnetic principles.

\subsection{Working Principle of Solenoid Coil}

A solid coil is made out of numerous turns of tightly wounded copper wire. When current passes through the coil, a strong magnetic field is created. Since the metal piston is magnetic, it reacts to the magnetic field created and moves upwards when electrical current is passed through the coil.

The direction of magnetic field is perpendicular to the direction of the flow of current. Consider the top of the coil as the north and the bottom as south. If the flow of current is from the north to the south terminal, the magnetic field also moves from north to south. The current in between the coil moves from south to north as the magnetic field is circulating. 


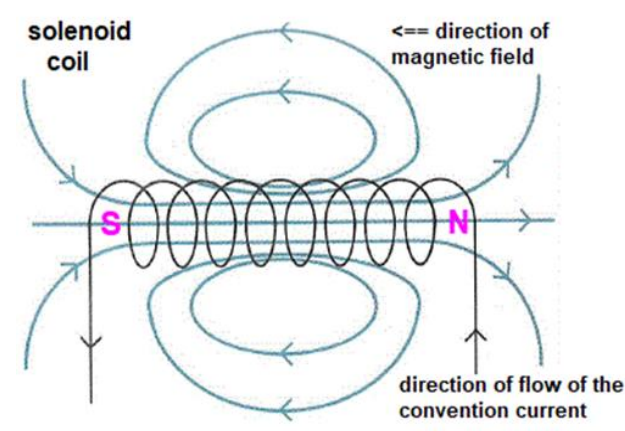

Fig.3. Working Principle of Solenoid Coil

\subsection{Working principle of the pistons}

The magnetic field in between the coils is moving from south to north. This causes the piston to move up. Once there is no more electrical current passing through the coil, the piston falls back to its original position due to gravity or in some cases with the aid of springs. The electrical current is passed and turned off to each coil in a specific sequence causing the pistons to also move up and down in a specific sequence.

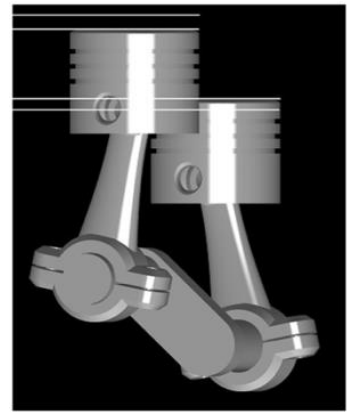

Fig.4. Pistons

\subsection{Working principle of crankshaft}

The pistons move in a linear motion and in a set sequence. They are connected to the crankshaft via a connecting rod. Being a 4-cylinder engine, the crankshaft has two crankpin and two connecting rods are connected on each crankpin. One crankpin is on an angle of 180 degree of the other. This specific position enables the crankshaft to rotate, therefore converting the linear motion from the pistons to the rotary motion of the crankshaft.

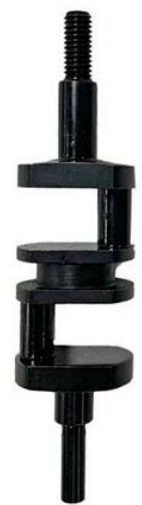

Fig.5. Crankshaft 


\section{Explanation in Detail}

The solenoid engine is a mechanism similar to that of a regular internal combustion. Instead of combustion in the cylinder causing the piston to move, solenoid engine has coils. These coils are typically a copper wire wounded tightly. Pistons inside the coils move by reacting to the magnetic field created by the coils when electrical charge is passed through it.

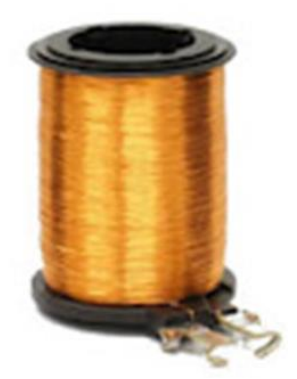

Fig.6. Copper Coil

When the electric charge is passed through the copper coil, it creates a magnetic field similar to that of a bar magnet. Just like a bar magnet, the copper coil has a north and south terminal. The direction of the magnetic field is perpendicular to the direction of the flow of current. When electric current is passed through the coil from north to south, the direction of the magnetic field inside the coil moves from south to north as the magnetic field is circulating. Unlike internal combustion engines, Solenoid engines produce a relatively lower power output. However, the power output of the solenoid engine can be increased by increasing the amount of current passing through the coil or increasing the number of turns in the coil. Doing so will increase the density of the magnetic field. This proves that the number of turns in the coil is directly proportional to the density of the magnetic field.

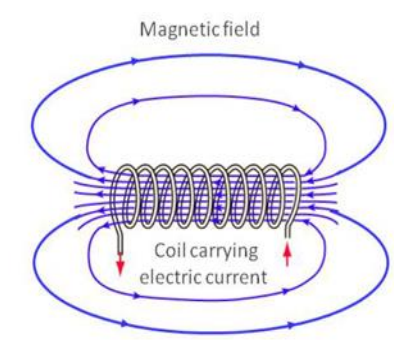

Fig.7. Illustration of Electromagnetic field

Solenoid engines can be created in many different ways and configurations. In this case, it is a V4 solenoid engine. V4 indicates that the engine has four cylinders/solenoids in a V configuration. As mentioned earlier, the solenoids initiate the physical motion in the engine by forming a magnetic field and resulting the piston to move in a linear form of motion. The engine is equipped with four pistons and is linked to the crankshaft with connecting rods.

Just like a combustion engine, the main goal of a solenoid engine is to rotate the crankshaft in order to produce output torque. This is only possible if the linear motion from the piston is converted to rotary motion at the crankshaft. The pistons are linked to the crankshaft using the connecting rods. A ball joint connects the crankshaft to the connecting rod. The ball joint allows free movements at angles. 
The crankshaft in this V4 solenoid engine has two crankpins. A crankpin is where the connecting rods connect to the crankshaft. Each crankpin has two connecting rods connected. First, the pistons on the right pushes the crankpin down, the piston on the left pulls the same crankpin up. It's the vice versa for the other crankpin. This process causes the crankshaft to rotate and creates output torque.

The solenoid causes the pistons to move and the pistons cause the crankshaft to rotate. When supplied with a constant amount of electric charge, the solenoid maintains a constant density of the magnetic field and the crankshaft rotates at a constant speed. The engine speed is measured in RPM (Rotations per Minute). It basically represents the amount of full rotations the crankshaft completes in the span of one minute. The faster the engine spins, the higher the RPM. The engine produces more power at higher RPMs as the engine is using up more electrical current and producing a greater output.

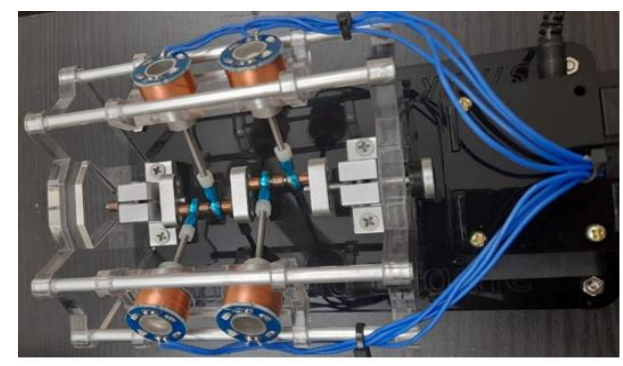

Fig.8. V4 Solenoid Engine

\section{Fabrication}

\subsection{Frame}

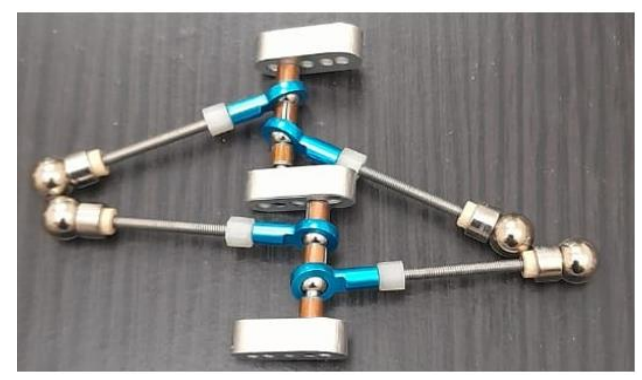

Fig.9. Frame

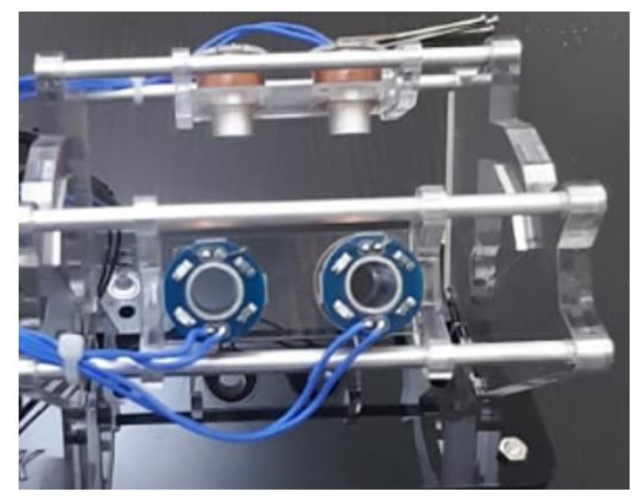

Fig.10. Shiny acrylic plactics to join the pieces together 
The project started by assembling the frame of the engine. The frame holds the engine in place and provides it a strong structure. The two transparent plastics were connected with the four metal rods. The black plastic base was then attached to the transparent ones using metal mounts. Shiny acrylic plastics contribute to the aesthetics of the project.

\subsection{Solenoids}

Then we proceeded to attach the four solenoid coils onto the frame structure. Four plastic mounts were attached in between the metal rods. The metal rods will firmly hold the plastic mounts in place. The solenoid coils were placed on to these plastic mounts. The plastic mounts will absorb a small amount of vibration while the engine is operating at high RPMs.

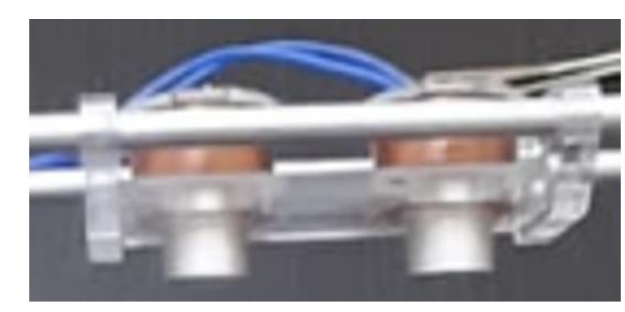

Fig.11. Solenoids

\subsection{Pistons and Connecting Rods}

Metal sphere pistons were then placed into the solenoid cylinders. Sphere shape will have minimum contact point with the cylinder walls compared to other shapes, hence reducing friction. We then glue the connecting rods to the sphere pistons from underside of the cylinders. Connecting rods will be spinning the crankshaft and at high RPMs resulting in greater stress on them. For this reason, connecting rods were chosen to be metal. Metal connecting rods will have minimum deflection compared to plastic ones, making the mechanism more robust.

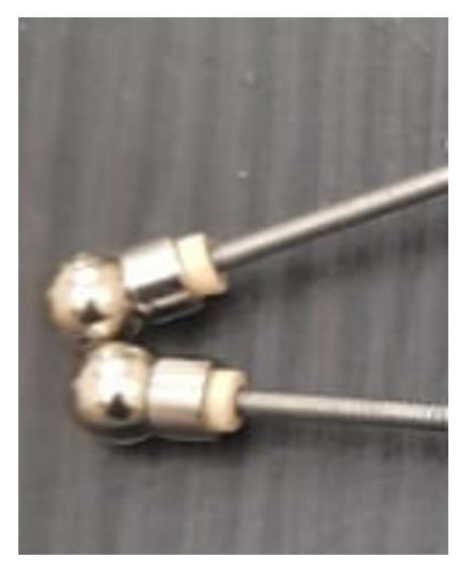

Fig.12. Pistons and Connecting Rods

\subsection{Crankshaft}

The crankshaft has two crankpins, each connects with two pistons on opposite sides. The crankshaft is made with small metal pieces joined together with metal bits that have holes to fit into them snugly. The crankshaft is linked with the connecting rods with ball joints to allow free movements in angles during the engine are operating. 


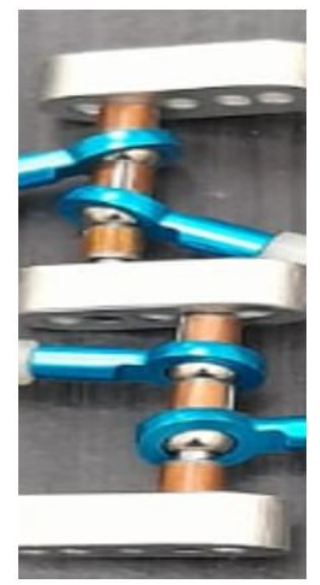

Fig.13. Crankshaft

\subsection{Circuit and casing}

Black acrylic plastics were glued together to create the box. The box was attached to the plastic base of the project. The glossy black color of the box matches with the base and compliments the aesthetics. A parallel circuit was created using wires, transformer, and a potentiometer. The parallel circuit powers the solenoids to create a magnetic field in order to operate the engine. The potentiometer controls the amount of voltage to control the engine's RPM. A knob was added to the potentiometer to make it easier to turn.

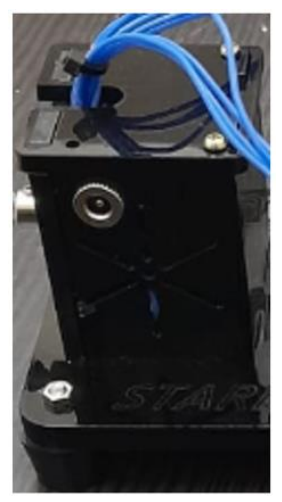

Fig.14. Casing for the circuit of the engine

\section{Results and Discussion}

\subsection{Results - Crank and piston performance}

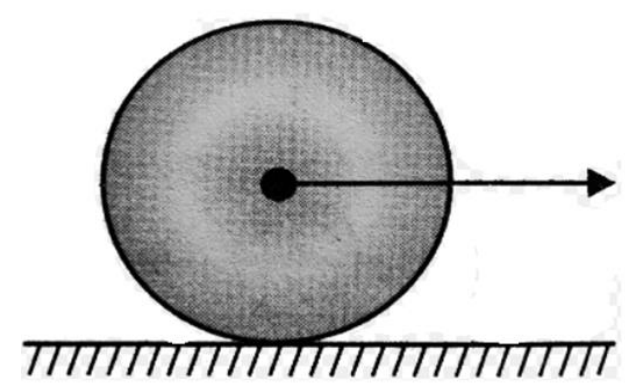

Fig.15. 
The solenoid engine is made from multiple components. The pistons and the crankshaft are made out of metal components. The pistons have a spherical shape to reduce the friction from in between the piston and the solenoid. This enables it to be able to handle high RPMs without any damage or noticeable deflections. The vibrations are minimum.

\subsection{Results - Solenoid Operation}

The solenoid coil in the engine is a type of wound coil solenoid. There are a lot of tight turns in the solenoid coil. This means, not a lot of current is required for the solenoid to create a magnetic field. The copper coil of the solenoid is wrapped tightly around the plastic cylinder. Inner surface of the tube is metallic and very smooth in order to allow the piston to slide back and forth with minimum resistance. It contributes to the smooth functioning of the engine and minimizes the vibrations.

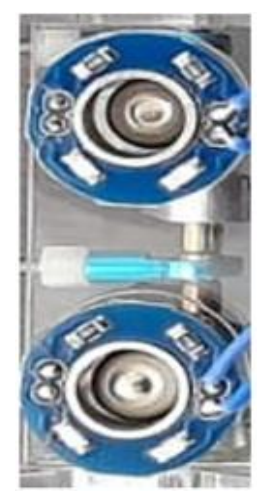

Fig.16.

\subsection{Discussion - Variable current flow}

As mentioned earlier, the solenoid coil has many folds. So many that it can be operated using minimal current. As a result, we added a potentiometer that controls the voltage provided to the solenoid. The current in the solenoid can be varied by twisting the knob. The solenoid will be operating at low voltage too, due to the fact that it has many folds on the copper coil contributing to create a magnetic field strong enough to move to pistons. Now the RPM of the engine is controllable by twisting of a knob. Twisting the knob allows more current to flow through the coil. Increasing the current passing through the solenoid will result in the engine spinning faster, therefore a higher RPM is obtained.

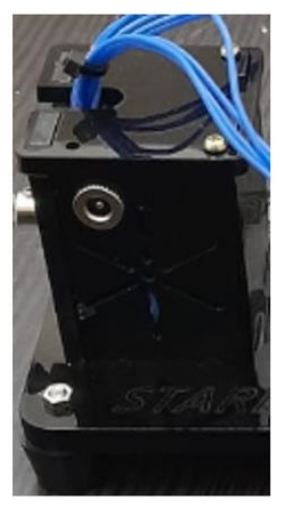

Fig.17. 


\subsection{Discussion - Aesthetics}

We placed the engine onto a black, acrylic plastic board. The glossy black base makes the engine stand out and appear more noticeable. Shiny metal parts of the engine mechanism also contribute to appear the project more aesthetic. We have also attached two transparent acrylic plastic pieces, one at the front and the other at the back of the engine. The pieces are shaped similar to the cross-section of the engine, giving it a neat appearance. The transparent plastics do not block the view of the mechanisms inside. Like all plastics, they will have quite a few deflections. When the engine is rotating at high RPMs, the plastics around will partially deflect and bend. These deflections will be minor enough to not be visible by naked eye. However, they will still have a role in absorbing the vibrations from the solenoid engine when operating, contributing to slightly quieter and refined operation.

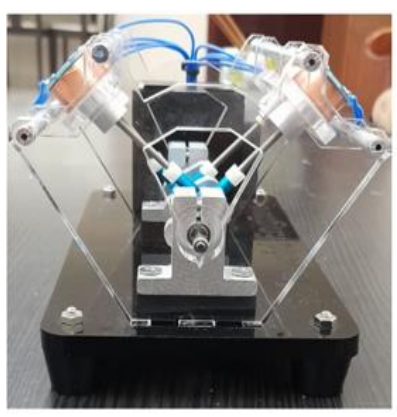

Fig.18.
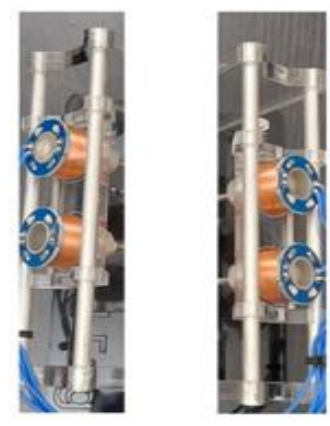

Fig.19.

Four metal rods connect the both transparent plastics on the front and back. It makes the engine look more robust and attractive while increasing the similarity with an actual internal combustion engine in terms of looks. Although the rods play a role in the aesthetics, in addition to making the project appear robust, it actually makes the project robust too. The metal rods are a part of the outer frame of the project and keep the plastics together, preventing them to move vigorously due to the vibrations coming from the engine operation. We chose blue wires to supply the electric current from the power supply to the solenoids. Blue wires complement electric and a futuristic theme of the project. The wires had been tucked away using zip ties to help make it appear neat.

\section{Conclusion and Future Works}

\subsection{Conclusions}

The solenoid engine operates as planned. It operates on the principles of electromagnetics. The pistons in the solenoids move in a linear motion and converts to rotary motion at the crankshaft. The current is supplied through a wall socket. The voltage from the socket creates a lot of electric charge in the solenoids. The charge creates a very dense magnetic field resulting in the engine to operate at a very high RPM. However, this issue has been resolved by varying the electrical charge to solenoids to control the RPM of the engine. However, another disadvantage using AC power source is that the solenoid engine does not operate when away from a wall socket or any other source of AC power supply.

\subsection{Future Works}

As mentioned previously, the solenoid engine will not operate without an AC power supply. This means that the 


\section{MJBAS}

Mediterranean Journal of Basic and Applied Sciences (MJBAS)

Volume 5, Issue 4, Pages 01-12, Oct-Dec 2021

solenoid engine needs to be around a source of AC power supply. AC power supplies are usually fixed points, resulting in limiting the portability of the project.

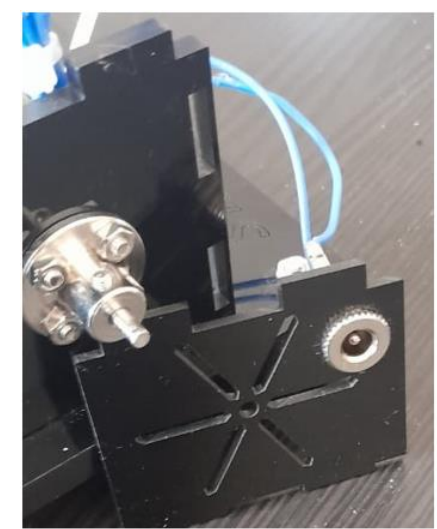

Fig.20.

To solve this issue, we need to switch from an AC power source to a DC power source. A rechargeable high voltage battery can replace an AC power source. The battery can be attached to the frame of the project and will be supplying electric charge to operate the solenoid. This solves the issue of portability of the solenoid engine. An AC power supply is no longer required to power the solenoid engine.

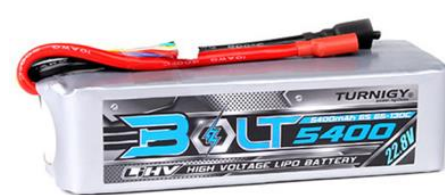

Fig.21.

\section{Declarations}

\section{Source of Funding}

This research did not receive any grant from funding agencies in the public, commercial, or not-for-profit sectors.

\section{Competing Interests Statement}

The authors declare no competing financial, professional and personal interests.

\section{Consent for publication}

Authors declare that they consented for the publication of this research work.

\section{References \\ [1] Lecture notes on Principals of Electronic Devices by Mr. Arumugam Ganesan \\ [2] [https://www.vedantu.com/formula/magnetic-field-in-a-solenoid-formula] \\ [3] [https://www.vedantu.com/physics/solenoid-engine]}


[4] [https://www.tlxtech.com/articles/solenoid-101-what-is-a-solenoid]

[5] [https://byjus.com/physics/solenoid-engine/]

[6] [https://www.rpi.edu/dept/phys/ScIT/InformationStorage/faraday/magnetism_a.html]

[7] [https://thepracticalengineer.com/blog/solenoidengine]

[8] [https://byjus.com/physics/solenoid-engine/]

[9] [https://en.wikipedia.org/wiki/Ferromagnetism]

[10] [https://www.britannica.com/science/ferromagnetism]

[11][https://www.khanacademy.org/science/physics/magnetic-forces-and-magnetic-fields/magnetic-field-current -carrying-wire/a/what-are-magnetic-fields] 